\title{
NOUVELLE
}

\section{Le RF9, un antagoniste puissant et sélectif des récepteurs du neuropeptide FF, prévient le développement de la tolérance aux opiacés}

Jean-Jacques Bourguignon, Guy Simonnet, Frédéric Simonin
J.J. Bourguignon, F. Simonin :

Institut Gilbert Laustriat, UMR 7175-LCl, CNRS,

Université Louis Pasteur,

boulevard Sébastien Brant, 67412 IIIkirch, France. simonin@esbs.u-strasbg.fr

G. Simonnet : Laboratoire Homéostasie-Allostasie-Pathologie, EA 3666, Université Victor Ségalen Bordeaux 2,

33076 Bordeaux, France.
> Les neuropeptides FF et AF sont deux peptides mammifères issus du même gène. Ils font partie de la famille des peptides RFamides qui présentent tous la séquence Arg-Phe- $\mathrm{NH}_{2}$ à leur extrémité carboxyterminale et ont comme cibles deux récepteurs couplés aux protéines $G$, appelés NPFFIR et NPFF2R [1]. Ces deux peptides ont été impliqués dans la régulation de nombreux processus physiologiques, comme la libération d'insuline, la prise alimentaire, la mémoire et la pression sanguine [2]. De plus, plusieurs études suggèrent que ces peptides sont impliqués dans le contrôle de la nociception et dans la modulation de l'analgésie induite par les opiacés [3, 4]. Les traitements chroniques par les opiacés produisent plusieurs effets secondaires, y compris le développement d'une tolérance aux effets analgésiques, qui conduit à augmenter les doses pour soulager la douleur. II a été envisagé que la désensibilisation et la dégradation (down regulation) des récepteurs opioïdes représenteraient l'une des bases moléculaires importantes sous-tendant ce phénomène. Une hypothèse alternative propose que la stimulation prolongée des récepteurs opioïdes déclencherait secondairement la stimulation de systèmes anti-opioïdes qui, en retour, provoquerait une hyperalgésie, diminuant ainsi l'effet initial de l'opiacé. Ce phénomène a été mis en évidence, in vivo, chez les rongeurs [5]. Chez l'homme, plusieurs publications indiquent que les traitements chroniques par les opiacés peuvent être associés à de I'hyperalgésie [6, 7]. Une augmentation de la sensibilité à la douleur a également été décrite chez les toxicomanes à l'héroïne [8]. Plusieurs systèmes neuromodulateurs, dont le NPFF, présentent des propriétés anti-opioïdes. Le NPFF, lorsqu'il est administré chez le rat, provoque de l'hyperalgésie qui s'oppose à l'analgésie morphinique. Cependant, l'absence d'antagonistes sélectifs des récepteurs du NPFF présentant une bonne stabilité métabolique et traversant la barrière hémato-encéphalique limitait fortement l'étude in vivo des fonctions de ce système. Lors d'une étude préliminaire, nous avions montré que le di-peptide benzoyl-ArgPhe- $\mathrm{NH}_{2}$ présentait une certaine affinité pour les récepteurs NPFF de la moelle épinière de rat. Afin d'identifier des ligands de haute affinité pour les récepteurs du NPFF, nous avons donc décidé de cribler une chimiothèque de dérivés du di-peptide Arg-Phe- $\mathrm{NH}_{2}$ par compétition sur le récepteur recombinant NPFF2R humain. Nous avons ainsi pu identifier un composé, le RF9 (Figure 1), qui présente une bonne affinité et une activité antagoniste pour ce récepteur. Ce composé s'est avéré présenter des caractéristiques similaires pour le récepteur NPFFlR humain. Par la suite, nous avons pu montrer que ce composé ne présentait pas ou peu d'affinité pour d'autres récepteurs couplés aux protéines $G$ proches, y compris les trois autres récepteurs mammifères de neuropep- tides RFamides connus à ce jour, GPR10, GPR54 et GPR103. Ces résultats indiquent donc une bonne sélectivité du RF9 pour les récepteurs du NPFF. Nous avons ensuite testé ce composé in vivo. Comme il avait été montré précédemment que l'injection intracérébroventriculaire de NPFF chez le rat provoquait une augmentation de la pression sanguine artérielle et du rythme cardiaque [9], nous avons examiné les effets du RF9 dans ce paradigme. Nous avons ainsi pu montrer que le RF9 n'a pas d'effet cardiovasculaire lorsqu'il est administré seul, mais qu'il bloque les effets sur la pression sanguine causée par le NPFF lorsqu'il est co-injecté avec celui-ci, confirmant ainsi son caractère antagoniste des récepteurs du NPFF. Des études précédentes montrant que le NPFF présentait des propriétés pronociceptives et anti-opioïdes, nous avons ensuite décidé d'évaluer la capacité du RF9

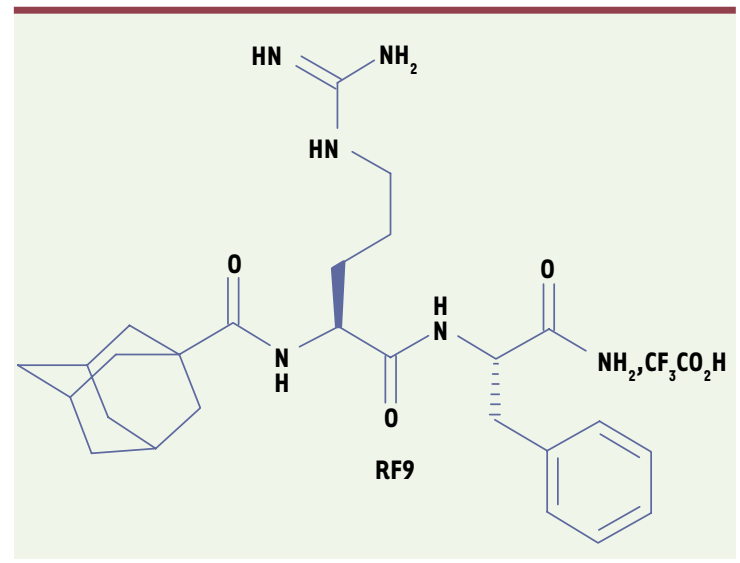

Figure 1. Structure du RF9. 
à prévenir le développement de l'hyperalgésie secondaire et de la tolérance associées à un traitement chronique par l'héroïne. Pour ce faire, nous avons choisi d'utiliser un modèle d'administration discontinue de dernier reflète mieux l'utilisation d'opiacés chez les patients souffrant de douleurs chroniques et par les toxicomanes [5]. Nous avons mesuré le seuil nociceptif basal faibles doses d'opiacé chez le rat, car ce

des animaux, à l'aide du test de vocalisation en réponse à une compression de la patte, au cours d'une co-administration par voie sous-cutanée de RF9 et d'héroïne pendant 14 jours (figure 2A). Dans cette même expérience, nous avons également mesuré l'effet analgésique de l'héroïne au premier et au dernier jour du traitement (figure 2B). Le RF9, lorsqu'il est co-administré avec l'héroïne, bloque complètement

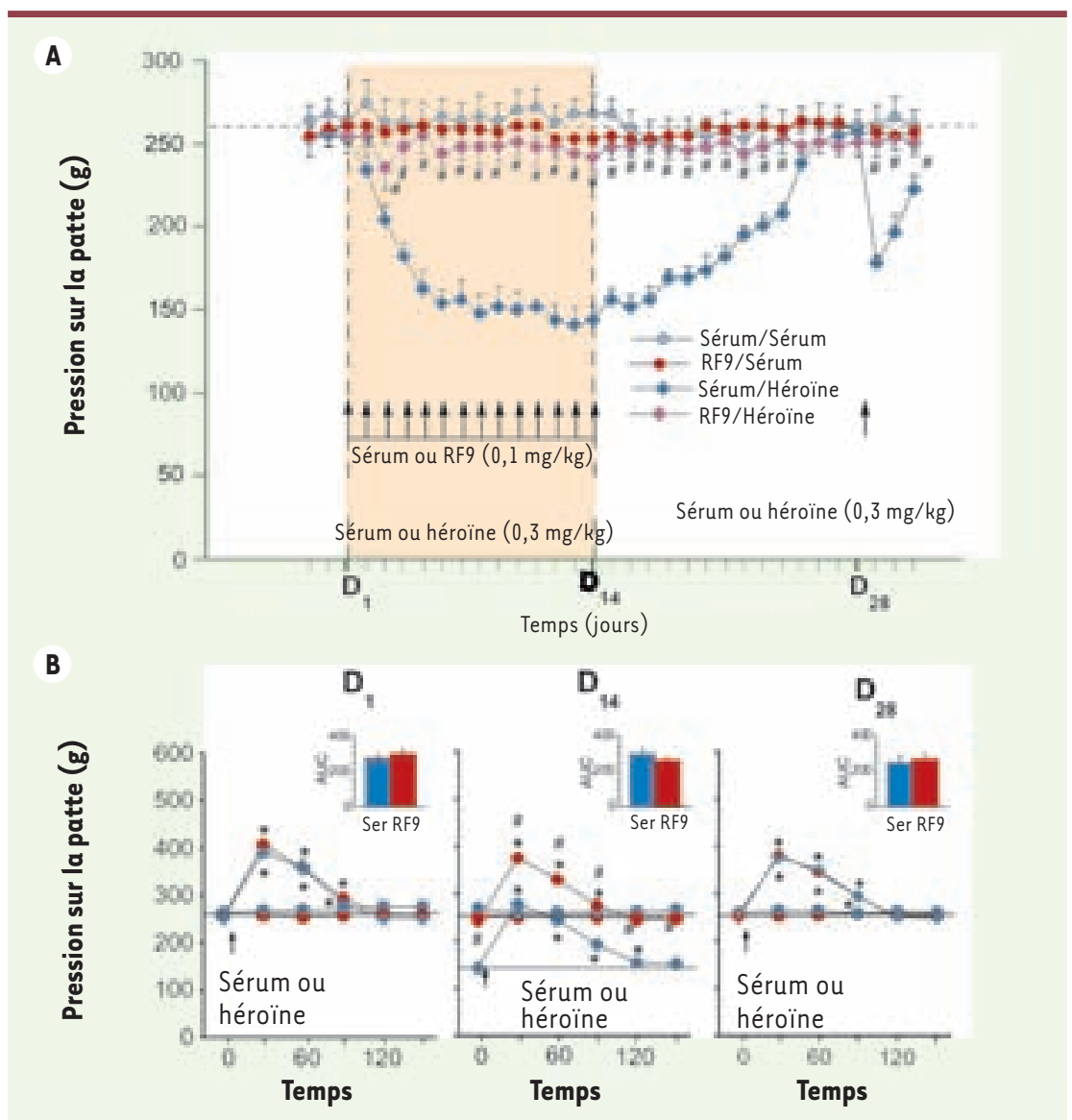

Figure 2. La co-administration du RF9 avec l'héroïne prévient l'hyperalgésie secondaire et la tolérance induites par l'héroïne. Les valeurs de pression de la patte déclenchant les vocalisations ( \pm SEM) sont exprimées en grammes. A. Effets de co-administrations de sérum physiologique ou de RF9 $(0,1 \mathrm{mg} / \mathrm{kg}) 30$ minutes avant l'administration de l'héroïne ou d'une solution de sérum physiologique sur le seuil nociceptif basal chez le rat $(n=8)$. Le seuil nociceptif basal a été déterminé une fois par jour 60 minutes avant chaque administration de sérum physiologique ou d'héroïne et après l'arrêt du traitement. $\# p<0,05$ avec le test de Newman-Keuls lorsque les groupes RF9/héroïne et sérum physiologique/héroïne ont été comparés. $B$. Effet analgésique induit par une injection d'héroïne $(0,3$ $\mathrm{mg} / \mathrm{kg}$ ) ou de sérum physiologique dans les différents groupes aux jours 1,14 et 28 . Le seuil nociceptif des animaux a été mesuré 30 minutes après l'injection, puis toute les 30 minutes jusqu'à la fin de l'effet pharmacologique. Le cartouche correspond à la comparaison des aires sous la courbe (AUC) pour les groupes sérum physiologique/héroïne et RF9/héroïne. ${ }^{\star} p<0,05$ avec le test de Dunnett en comparaison avec les valeurs de seuil nociceptif basal; \# $p<0,05$ avec le test de Newman-Keuls lorsque les groupes RF9/héroïne et sérum physiologique/héroïne ont été comparés. l'hyperalgésie de longue durée faisant suite à l'effet analgésique de chaque administration d'héroïne et le développement de la tolérance à l'effet analgésique de l'opiacé. Administré seul, ce composé n'a aucun effet sur le seuil nociceptif des animaux. Ces résultats confirment pleinement le rôle anti-opioïde du système NPFF et démontrent que le blocage des récepteurs NPFF peut prévenir efficacement l'hyperalgésie secondaire induite par les opiacés, et par voie de conséquence la tolérance associée.

Nos résultats renforcent l'idée que le développement de la tolérance aux effets analgésiques des opiacés n'est pas seulement dû à une diminution de la réponse cellulaire liée à la désensibilisation et/ou à la down regulation des récepteurs opioïdes, mais qu'il pourrait également avoir pour origine une activation secondaire de systèmes anti-opioïdes (comme le NPFF) qui conduirait à une augmentation durable de la sensibilité à la douleur. De plus, cette étude suggère que le blocage sélectif des récepteurs du NPFF à l'aide d'antagonistes pourrait représenter une stratégie innovante pour accroître l'efficacité des opiacés dans le cadre du traitement des douleurs chroniques [10]. $\diamond$ RF9, a powerful and selective antagonist of the neuropeptide FF receptors, prevents the development of the tolerance to opioids

\section{RÉFÉRENCES}

1. Zajac JM. Neuropeptide FF: new molecular insights. Trends Pharmacol Sci $2001 ; 22$ : 63.

2. Panula P, Aarnisalo AA, Wasowicz K. Neuropeptide FF, a mammalian neuropeptide with multiple functions. Prog Neurobiol 1996 ; 48 : 461-87.

3. Roumy M, Zajac JM. Neuropeptide FF, pain and analgesia. EurJ Pharmacol $1998 ; 345: 1-11$.

4. Panula $P$, Kalso $\varepsilon$, Nieminen $M$, et al. Neuropeptide FF and modulation of pain. Brain Res 1999 ; 848 : 191-6.

5. Celerier $\varepsilon$, Laulin JP, Corcuff JB, et al. Progressive enhancement of delayed hyperalgesia induced by repeated heroin administration: a sensitization process. J Neurosci $2001 ; 21: 4074-80$.

6. Simonnet G, Rivat C. Opioid-induced hyperalgesia: abnormal or normal pain ? NeuroReport 2003 ; $14: 1-7$.

7. Angst MS, Clark JD. Opioid-induced hyperalgesia: a qualitative systematic review. Anesthesiology 2006 ; $104: 570-87$.

8. White JM. Pleasure into pain: the consequences of longterm opioid use. Addict Behav 2004 ; 29 : 1311-24.

9. Jhamandas JH, MacTavish D. Central administration of neuropeptide FF causes activation of oxytocin paraventricular hypothalamic neurones that project to the brainstem.J Neuroendocrinol 2003 ; 15 : 24-32.

10. Simonin F, Schmitt M, Laulin JP, et al. RF9, a potent and selective neuropeptide $F F$ receptor antagonist, prevents opioid-induced tolerance associated with hyperalgesia. Proc Natl Acad Sci USA 2006 ; 103 : 466-71. 